\title{
Chromosome 15q11-q13 copy number gain detected by array-CGH in two cases with a maternal methylation pattern
}

\author{
Ee-Shien Tan ${ }^{1}$, Min-Hwee Yong ${ }^{2}$, Eileen CP Lim³ ${ }^{3}$, Zhi-hui Li ${ }^{4}$, Maggie SY Brett ${ }^{3}$ and Ene-Choo Tan ${ }^{3,5^{*}}$
}

\begin{abstract}
Background: The 15q11-q13 region contains many low copy repeats and is well known for its genomic instability. Several syndromes are associated with genomic imbalance or copy-number-neutral uniparental disomy. We report on two patients: Patient 1 is a boy with developmental delay and autism; and Patient 2 is a girl with developmental delay, hypotonia and dysmorphism. We performed analyses to delineate their dosage in the $15 q$ region, determine whether the patients' dosage correlates with phenotypic severity, and whether genes in the amplified regions are significantly associated with identified functional networks.
\end{abstract}

Results: For the proximal region of $15 q$, molecular cytogenetic analysis with Agilent oligonucleotide array showed a copy number of 3 for Patient 1 and a copy number of 4 for Patient 2. Fluorescent in situ hybridization analysis of Patient 2 showed two different populations of cells with different marker chromosomes. Methylation analysis of the amplified region showed that the extra copies of small nuclear ribonucleoprotein polypeptide $\mathrm{N}$ gene were of maternal origin. Phenotypic severity did not correlate with the size and dosage of $15 q$, or whether the amplification is interstitial or in the form of a supernumerary marker. Pathway analysis showed that in Patient 2 , the main functional networks that are affected by the genes from the duplicated/triplicated regions are developmental disorder, neurological disease and hereditary disease.

Conclusions: The 15q11-q13 gains that were found in both patients could explain their phenotypic presentations. This report expands the cohort of patients for which 15q11-q13 duplications are molecularly characterized.

Keywords: 15q duplication syndrome, Array comparative genomic hybridization (aCGH), Copy number gain, Autism, Developmental delay, Fluorescence in situ hybridization (FISH), Marker chromosome

\section{Background}

The 15q11-q13 region is a hotspot for recombination. Several breakpoint (BP) regions have clusters that contain low copy repeats and segmental duplications [1]. Chiasmata frequency in the region is known to be higher than in other chromosomal regions [2]. As a consequence, the region is prone to having deletions, duplications and rearrangements. In addition to frequent genomic rearrangements, this chromosomal region is also highly regulated by methylation, and the allele that is expressed for specific

\footnotetext{
* Correspondence: tanec@bigfoot.com

${ }^{3}$ KK Research Laboratory, KK Women's \& Children's Hospital, 100 Bukit Timah Road 229899 Singapore, Singapore

${ }^{5}$ Office of Clinical Sciences, Duke-NUS Graduate Medical School, 8 College

Road 169857 Singapore, Singapore

Full list of author information is available at the end of the article
}

genes is based on the parental origin of the chromosome. The presence of imprinted genes in this region means that there may be phenotypic consequences even if the rearrangements are copy number neutral and results in no genomic imbalance.

Genomic disorders mapped to this region include Angelman syndrome (Online Mendelian Inheritance in Man (OMIM) \#105830) and Prader-Willi syndrome (OMIM \#176270). In the majority of cases, both of the syndromes are due to either deletion or uniparental disomy. Of the remaining cases of Angelman syndrome, approximately $10-15 \%$ are due to $U B E 3 A$ mutations and $2-4 \%$ are due to imprinting centre defect. Less than $1 \%$ of the remaining Prader-Willi syndrome cases are due to imprinting centre defect [3]. Cytogenetically visible duplications (OMIM \#608636) and marker chromosomes from 
derivatives of this region are also common. The high frequency of such cases gave rise to a clinically recognizable disorder called 15q duplication syndrome, with some common neurobehavioural phenotypes [4]. Copy numbers of three, four, five and six have all been reported for this region [5-7].

Most copy number gains in this region are due to translocations, inversions and supernumerary marker chromosomes (sSMC). Interstitial duplications/triplications and balanced translocations (which do not result in copy number changes) are more infrequent [8]. To add to the clinical phenotype of $15 q$ duplication cases, we present two patients - a Malay boy with partial trisomy $15 \mathrm{q}$ and a Chinese girl with mosaic partial tetrasomy $15 \mathrm{q}$. We conducted analyses to delineate their amplification in the $15 \mathrm{q}$ region, analyze the region at the gene level, and determine whether genes within the amplified regions are significantly associated with identified functional networks.

\section{Presentation of cases}

The two patients described in this study were participants in a study to identify genomic imbalance in patients with developmental delay/multiple congenital anomalies. They were recruited from the genetics outpatient clinics of the KK Women's and Children's Hospital, Singapore. The study was approved by the SingHealth Institutional Review Board, which oversees all research studies in the hospital. The patients were recruited with the written informed consent of their parents.

Patient 1 is the $6^{\text {th }}$ child of healthy, unrelated parents of Malay ancestry. His mother was 33 years old at the time of his birth. He has an older brother with autism spectrum disorder, but four other siblings are phenotypically normal. He was delivered at full term with a birth weight of $2950 \mathrm{~g}$ and there were no perinatal issues. He first presented at 5 years and 8 months of age with severe language delay, hyperactivity and a preoccupation with water. His verbal language was limited to the repetition of a few words and pointing for needs. His teachers reported that it was difficult to engage him in his pre-school activities. He was diagnosed with autism spectrum disorder and intellectual impairment. Clinical examination revealed a well thrived child with no dysmorphic features. Fragile X testing result was normal.

The second patient was a girl of Chinese descent born at 39 weeks gestation with a birth weight of $3234 \mathrm{~g}$, length of $45.5 \mathrm{~cm}$ and head circumference of $32 \mathrm{~cm}$. Her Apgar scores at 1 and 5 minutes were both 9. She is the third child of a non-consanguineous marriage with no family history of autism or learning impairment. Her mother was 28 years old and her father 29 years old at the time of her birth. She was first noted to have gross motor delay at 9 months of age. The initial investigations, including a thyroid function test and metabolic screen, were normal. On further review, she continued to have significant developmental delay. At two years of age, she was able to walk with support and she spoke no clear words. Assessment using the Age and States Questionnaire, Third Edition, showed normal scores for fine motor and personal-social skills, and a borderline score in communication skills. The patient had significant delays in gross motor and problem-solving skills.

On physical examination, her height and weight were at the $90^{\text {th }}$ percentile and her head circumference was at the $97^{\text {th }}$ percentile. She had truncal hypotonia and pigmentation, irregular pigmentation on her lower limbs, protruding tongue and hypertelorism, but no other dysmorphic features. Chromosome culture revealed an abnormal female karyotype, with two cell lines that showed a different additional marker chromosome in each cell line.

\section{Results}

\section{Karyotype analysis}

Karyotype information on Patient 1 was unavailable. For Patient 2, karyotype is reported as $47, \mathrm{XX},+$ mar.ish del (15)(q12)(SNRPN+,D15Z1+)[14]/47,XX,+mar.ish psu dic $(15 ; 15)($ SNRPN++,D15Z1++) [4]. Two different markers were identified. The larger marker chromosome was found in 14 out of 18 metaphases analyzed. Fluorescent in situ hybridization (FISH) analysis showed two centromeric 15 signals (D15Z1) and two signals localized to SNRPN on this marker chromosome (Figure 1A). A smaller marker chromosome was found in the other 4 metaphases. FISH analysis on this smaller marker chromosome showed one centromeric 15 signal and one signal localized to SNRPN (Figure 1B). Hence, cytogenetic and FISH analysis showed that this patient is mosaic for the two marker chromosomes, with 14 out of 18 cells harboring the marker chromosome with two copies of proximal $15 \mathrm{q}$ and the remaining 4 cells harboring the marker chromosome with one copy of proximal $15 \mathrm{q}$, corresponding to partial tetrasomy and partial trisomy 15q.

\section{Array-based copy number analysis}

For Patient 1, analysis showed a gain in copy number from $21,213,950$ to $26,208,646$, involving 710 probes with a mean $\log _{2}$ ratio of 0.5585 for the proximal region of the long arm of chromosome 15 (Figure 2A). For Patient 2, the gain was from $18,362,555$ to $26,208,646$, involving 865 probes with mean $\log _{2}$ ratio of 1.0239 (Figure 2B). From the position of the probes, the estimated minimum size of the gain for Patient 1 was 4,994,696 basepairs (bp) while the maximum size was $6,045,492 \mathrm{bp}$. For Patient 2, the minimum size for the gain was $7,846,091 \mathrm{bp}$. The maximum size could not be determined as the gain started from the first probe for the chromosome. The list of known genes in the amplified region is shown in Table 1. 

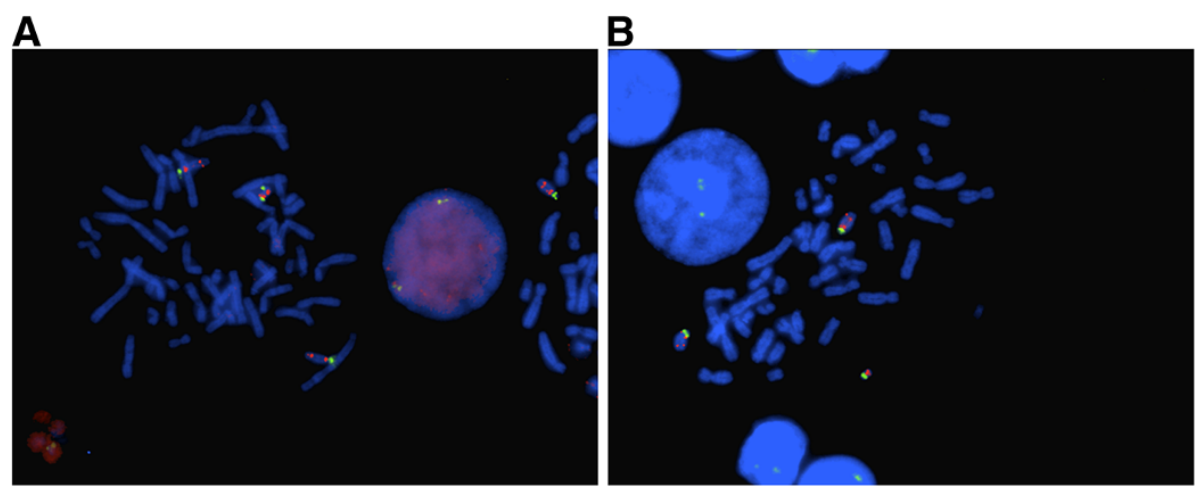

Figure 1 FISH analysis of patient 2. (A) 2 green and 2 red hybridization signals were seen on the larger marker corresponding to centromeric 15 (D15Z1) and SNRPN respectively (B) 1 green and 1 red hybridization signals were seen on the smaller marker corresponding to centromeric 15 (D15Z1) and SNRPN respectively (Abbreviations: FISH: Fluorescent in situ hybridization, SNRPN: Small nuclear ribonucleoprotein polypeptide N).

\section{Quantitative polymerase chain reaction analysis}

Gene copy number was also investigated using relative quantitative real-time polymerase chain reaction (qRTPCR) with SYBR Green dye and SNRPN as the target for quantifying copy number. Analysis showed copy number of 1.604 for SNRPN relative to the reference gene for Patient 1 and 2.098 for Patient 2 when compared against a phenotypically normal control. This corresponds to 3 copies and 4 copies, respectively (Figure 3A).

\section{Methylation analysis}

Sodium bisulfite treatment followed by qRT-PCR showed that both the methylated and unmethylated SNRPN alleles were present. Patient 1 had two methylated alleles and
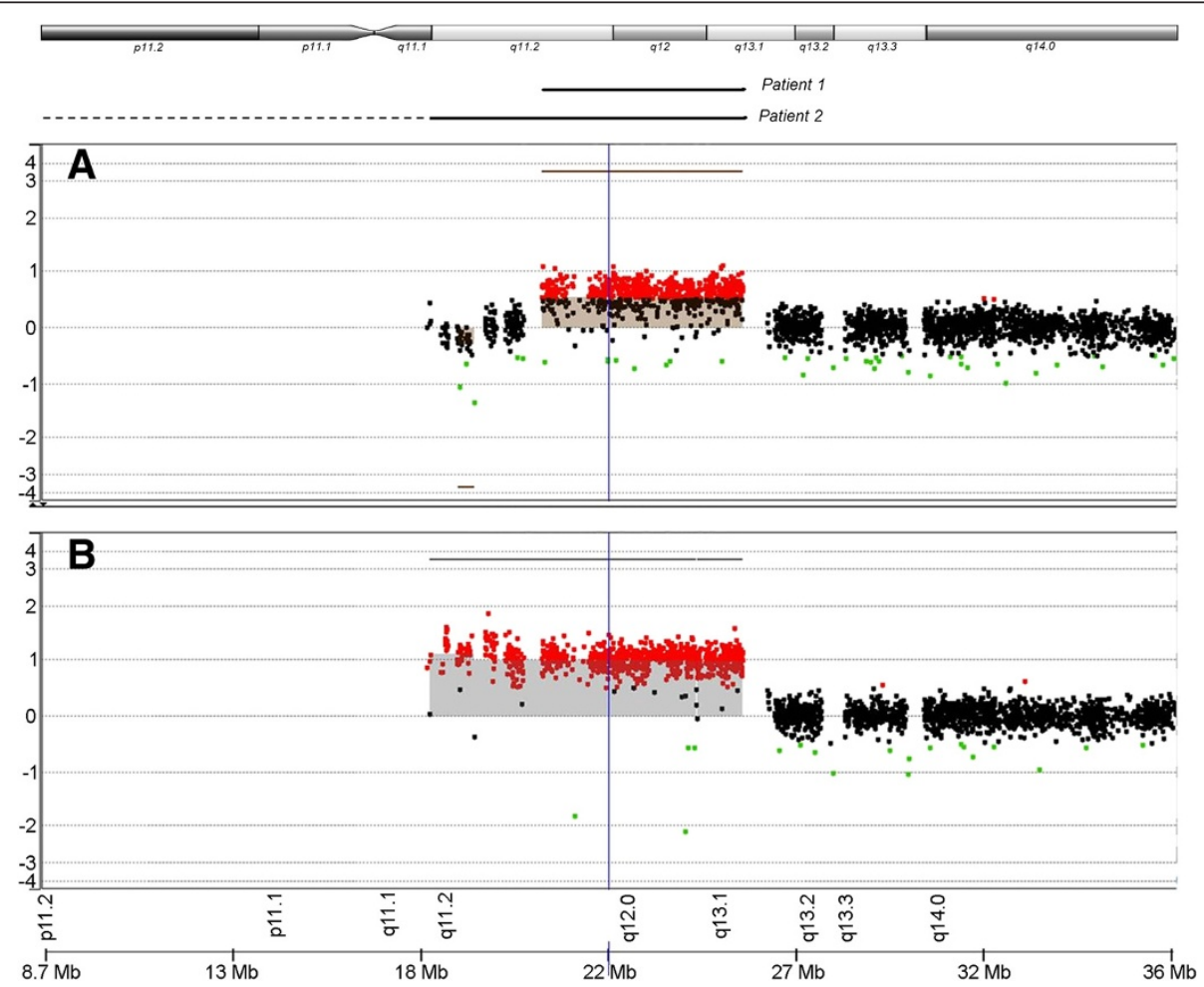

Figure 2 Screenshot of analysis with Genomic Workbench Lite for Array-CGH results. Chart shows the gain in copy number from $15 q 11$ to $15 q 13$ for (A) Patient 1 and (B) Patient 2. The regions with the copy number gain corresponding to the CGH results were indicated by the solid lines below the ideogram. (Abbreviations: CGH; Comparative genomic hybridization; Mb: million base-pairs). 
Table 1 List of genes for the duplicated region for patient 2

\begin{tabular}{|c|c|c|c|c|c|}
\hline Symbol & Gene name & $\mathrm{HI}(\mathrm{D})^{1}$ & $\mathrm{HI}\left(\mathrm{I}^{2}\right.$ & $\mathrm{TS}(\mathrm{I})^{3}$ & Imprinting $^{4}$ \\
\hline POTEB & POTE ankyrin domain family, member B & - & - & - & - \\
\hline OR4M2 & Olfactory receptor, family 4 , subfamily $M$, member 2 & 93.7 & - & - & - \\
\hline OR4N4 & Olfactory receptor, family 4 , subfamily $\mathrm{N}$, member 4 & 78.3 & - & - & - \\
\hline GOLGA6LI & Golgin A6 family-like 1 & - & - & - & - \\
\hline TUBGCP5 & Tubulin-gamma complex-associated protein 5 & - & - & - & - \\
\hline CYFIP1 & Cytoplasmic FMRP interacting protein 1 & 53.3 & - & - & - \\
\hline NIPA2 & Not imprinted in Prader-Willi syndrome/Angelman syndrome 2 & 52.9 & 0 & 0 & - \\
\hline NIPA ${ }^{*}$ & Not imprinted in Prader-Willi syndrome/Angelman syndrome 1 & 25.3 & 0 & 0 & - \\
\hline GOLGA6L2 & Golgin A6 family-like 2 & - & - & - & - \\
\hline MKRN3 & Makorin ring finger protein 3 & 98.7 & 0 & 0 & $P$ \\
\hline MAGEL2 & MAGE-like 2 & - & - & - & $P$ \\
\hline$N D N^{*}$ & Necdin, melanoma antigen (MAGE) family member & 36.2 & - & - & $\mathrm{P}$ \\
\hline$N P A P 1^{*}$ & Nuclear pore associated protein 1 & 93.6 & - & - & - \\
\hline$S N R P N^{*}$ & Small nuclear ribonucleoprotein polypeptide $\mathrm{N}$ & 11.3 & - & - & $P$ \\
\hline SNURF & SNRPN upstream reading frame & 64.6 & - & - & $P$ \\
\hline$U B E 3 A^{*} \wedge$ & Ubiquitin protein ligase E3A & 23.2 & 3 & 0 & M \\
\hline ATP10A* & ATPase, class V, type 10A & 74.0 & 0 & 0 & M \\
\hline GABRB3* & Gamma-aminobutyric acid (GABA) A receptor, beta-3 & 5.2 & 0 & 0 & - \\
\hline GABRA5 & Gamma-aminobutyric acid (GABA) A receptor, alpha-5 & 25.5 & - & - & - \\
\hline GABRG3 & Gamma-aminobutyric acid (GABA) A receptor, gamma-3 & 83.8 & - & - & - \\
\hline OCA2* & Pink-eye dilution, murine, homolog of (oculocutaneous albinism II) & 71.5 & 0 & 0 & - \\
\hline HERC2* & HECT domain and RCC1-like domain 2 & 42.7 & - & - & - \\
\hline
\end{tabular}

Notes: Gene list and names according to Decipher (https://decipher.sanger.ac.uk/). The list for Patient \#1 is from MKRN3 to HERC2.

*OMIM morbid genes.

$\wedge$ Associated with developmental disorders according to the Developmental Disorders Genotype-Phenotype Database (DDG2P).

${ }^{1} \mathrm{HI}(\mathrm{D})$ Haploinsufficiency scores from Decipher: values from 0-100\%. Low values (0-10\%) indicate more likely to exhibit Haploinsufficiency.

${ }^{2} \mathrm{HI}(\mathrm{I})$ ISCA Haploinsufficiency Score $(0=$ no evidence available, $1=$ Little evidence, $2=$ Some evidence, $3=$ Sufficient evidence for dosage pathogenicity).

${ }^{3} \mathrm{TS}(\mathrm{I})$ ISCA Triplosensitivity Score $(0=$ no evidence available, $1=$ Little evidence, $2=$ Some evidence, 3 = Sufficient evidence for dosage

pathogenicity) (http://www.ncbi.nlm.nih.gov/projects/dbvar/ISCA/).

${ }^{4}$ For last column: $\mathrm{M}=$ expressed from maternal allele, $\mathrm{P}=$ expressed from paternal allele.

Abbreviations: FMRP; Fragile X mental retardation protein; HECT: Homologous to the E6-AP carboxyl terminus; SNRPN; small nuclear ribonucleoprotein polypeptide $\mathrm{N}$.

one unmethylated allele. For patient 2, the copy number of the methylated allele was three times the unmethylated one when compared with the control sample (Figure 3B).

\section{Pathway analysis}

Ingenuity Pathway Analysis (IPA) using genes from the duplicated/triplicated region for Patient 2 identified developmental disorder, neurological disease and hereditary disease as the main networks affected. The broad categories of the pathways with their statistically significant $p$-values are presented in Table 2. Enrichment analysis for diseases and biological functions was performed on the constructed networks. Those with at least five molecules in the network are presented in Table 3.

\section{Discussion}

Although duplications that involve the proximal region of $15 \mathrm{q}$ are one of the most common rearrangements in pediatric patients with congenital disorders, only a few megabase cases have been mapped by molecular karyotyping or analyzed at the gene level. Of the duplications identified by molecular methods, the sizes ranged from about 1 million base-pairs $(\mathrm{Mb})$ in a multiplex ligationdependent probe amplification study [9] to 17.7 Mb using CGH arrays with 244 $\mathrm{K}$ oligonucleotide probes [10]. A few large studies have found recurrent microdeletions or duplications in the 15q11-q13 region for idiopathic epilepsies, autism, and combined schizophrenia and epilepsy [11-16]. There is another relevant region for psychiatric disorders that is more distal, with CHRNA7 (cholinergic receptor, nicotinic subunit alpha 7) within the BP4-BP5 region as the top candidate gene [17-19].

In a large study using published data sets, the frequency of $15 \mathrm{q}$ duplications is reported to be 1:494 for autism cohorts and 1:508 for clinical cohorts with intellectual disability, ASD, or multiple congenital anomalies [20]. The 


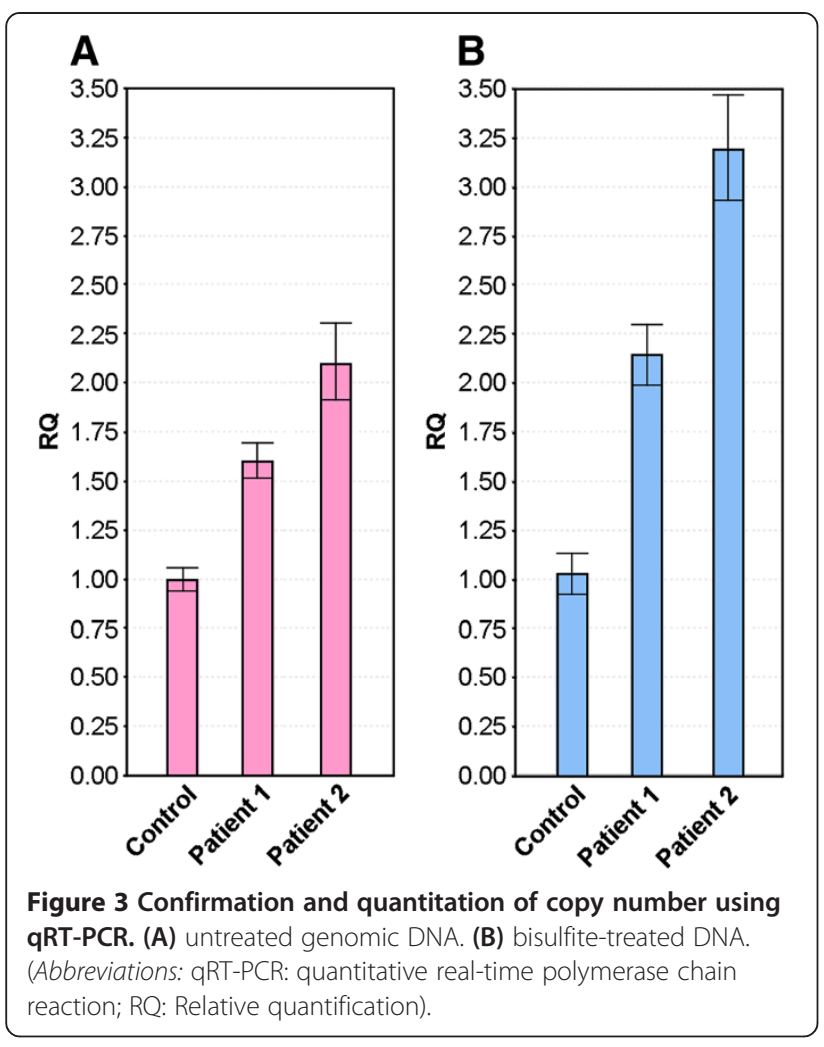

two patients with $15 \mathrm{q}$ duplication/triplication in this report were from 350 cases with developmental delay and/ or multiple congenital anomalies who were prospectively recruited into our array-CGH study. The gains in the two patients had different proximal breakpoints. They appeared to share the same distal breakpoint, which is within a segmental duplication that corresponds to the BP3 region, ending after the last three exons of the HERC2 (HECT domain and RCC1-like domain 2) which are duplicated.

No karyotype data was available for Patient 1, who has Class II duplication [21]. The duplication is likely to be an interstitial microduplication with the proximal breakpoint located within BP2. The first duplicated sequence with potential function is microRNA 4508 (gain of one copy). At least 14 genes are in the duplicated region (13 OMIM genes and 8 OMIM morbid genes). The first gene duplicated is MKRN3 (Makorin ring finger protein 3).

The triplication in Patient 2 is in the form of an SSMC. We could not ascertain the start of the copy number gain as there was no array-CGH probe for $15 \mathrm{p}$ and the gain started before the recognized $\mathrm{BP} 1$ region and the first known gene (CHEK2P2), which is a pseudogene on $15 q$. If it was interstitial and the start was near the first array CGH probe, it would be a Class I duplication [21] with the proximal and distal breakpoints within BP1 and BP3, respectively. Alternatively, it could involve the whole $\mathrm{p}$ arm. Array-CGH did not detect any other genomic imbalance for this patient. The gain involves at least 22 genes out of which 18 are OMIM genes and 9 are OMIM morbid genes (Table 1). At least three genes within this region (NIPA1, NIPA2, CYFIP1) are implicated in the development of the central nervous system while a fourth gene, TUBGCP5, is a member of the cytoskeleton tubulin complex in cells and is evolutionarily conserved [22].

Although parental samples were not available for both patients, qRT-PCR analysis using primers which are specific for methylation states showed that the extra chromosomal material for both patients had methylation pattern that implied gains of maternal origin. This is consistent with the maternal origin of such duplications being more common, and with such duplications being more likely to have pathogenic consequences. Gain in copy number of maternally-derived SNRPN has been associated with autism [22], but Patient 2 had not presented with such features. One notable feature is that her physical dimensions were above the $90^{\text {th }}$ percentile. Overgrowth has been reported in patients with an increased dosage of distal 15q [23], but this patient's copy number gain does not involve the distal $15 \mathrm{q}$ region.

For the two patients in this report, phenotypic severity did not correlate with the size and dosage of the distal breakpoint on $15 \mathrm{q}$, and whether the duplication is interstitial or in the form of an sSMC. This lack of correlation could be due to additional factors such as presence of mosaicism in Patient 2, genetic background, epigenetic modifications, and gender. Aside from the increase in gene dosage and the parental origin of the duplicated genes, additional alterations at the epigenetic level could influence gene expression, which could lead to phenotypic variability for patients who carry duplications of the same size and dosage [21,24]. Hogart et al. provided some supporting evidence when they measured the level of 10 transcripts within the 15q11-13 region in two postmortem brains and found that the expression pattern correlated with parental gene dosage in the male patient. In the female brain, there was decreased expression of SNRPN, $N D N$, small nuclear RNAs (snoRNAs) and gammaaminobutyric acide $(G A B A)_{A}$ despite an increased dosage of genes of maternal origin [25]. In the case of SNRPN, the decreased expression was consistent with the finding of increased methylation found at the imprinting control region.

Mosaicism is common in $15 \mathrm{q}$ duplications that involve supernumerary derivative chromosome 15 . Such duplications tend to take the form of pseudodicentric derivative chromosomes rather than intrachromosomal. The pseudodicentric marker chromosome, psu dic $(15 ; 15)$, is usually formed by a homologous recombination between two chromosomes 15 [26]. The smaller marker chromosome in Patient 2 is likely to be the result of a break in the psu $\operatorname{dic}(15 ; 15)$, before the inactivation of one centromere 
Table 2 List of systems/processes associated with genes in the amplified region as identified by IPA

\begin{tabular}{|c|c|c|}
\hline Category & $p$-values & Molecules encoded from duplicated region \\
\hline Auditory and Vestibular System Development and Function & 2.20E-03 & GABRA5,GABRB3 \\
\hline Auditory Disease & $2.20 \mathrm{E}-03-8.41 \mathrm{E}-03$ & GABRA5,GABRB3 \\
\hline Behaviour & $1.36 \mathrm{E}-03$ & GABRB3 \\
\hline Cancer & 1.07E-02-4.42E-04 & ATP10A,MKRN3,SNRPN,UBE3A \\
\hline Cardiac Arteriopathy & 4.33E-03 & GABRA5,GABRB3,GABRG1,GABRG3 \\
\hline Cardiovascular Disease & 4.33E-03-4.44E-08 & GABRA5,GABRB3,GABRG1,GABRG3 \\
\hline Cell cycle & $1.02 \mathrm{E}-02-4.42 \mathrm{E}-04$ & HERC2, UBE3A \\
\hline Cell Death and Survival & $3.03 \mathrm{E}-03-1.17 \mathrm{E}-03$ & GABRA5,GABRB3 \\
\hline Cell Morphology & $2.68 \mathrm{E}-04-1.08 \mathrm{E}-02$ & OCA2, GABRA5,GABRB3,NDN \\
\hline Cell-To-Cell Signalling and Interaction & $1.57 \mathrm{E}-01-8.12 \mathrm{E}-03$ & NDN, UBE3A \\
\hline Cellular Assembly and Organization & $1.02 \mathrm{E}-02-7.54 \mathrm{E}-03$ & SNURF \\
\hline Cellular Compromise & $3.36 \mathrm{E}-05-1.72 \mathrm{E}-03$ & GABRA5,GABRB3 \\
\hline Cellular Development & 8.05E-03-3.36E-05 & NDN, UBE3A \\
\hline Cellular Growth and Proliferation & 8.05E-03-5.96E-04 & NDN, UBE3A \\
\hline Cellular Movement & $2.68 \mathrm{E}-04-1.51 \mathrm{E}-04$ & GABRA5,GABRB3,NDN \\
\hline Connective Tissue Development and Function & 1.57E-01 & NDN \\
\hline Connective Tissue Disorders & $1.40 \mathrm{E}-03-1.40 \mathrm{E}-08$ & GABRA5,GABRB3,GABRG3 \\
\hline Developmental Disorder & 1.17E-02-4.69E-10 & GABRB3,GABRG3,MAGEL2,MKRN3,NDN,SNRPN,UBE3A \\
\hline DNA Replication, Recombination, and Repair & 1.15E-02 & SNRPN \\
\hline Endocrine System Disorders & 8.57E-03 & MKRN3 \\
\hline Gene Expression & $1.15 \mathrm{E}-02-6.35 \mathrm{E}-05$ & NDN,SNRPN,UBE3A \\
\hline Hair and Skin Development and Function & 4.97E-03 & OCA2 \\
\hline Hematological Disease & 5.83E-03 & UBE3A \\
\hline Hepatic System Development and Function & 1.57E-01 & NDN \\
\hline Hereditary Disorder & 1.17E-02-4.69E-10 & GABRA5,GABRB3,GABRG3,MAGEL2,MKRN3,NDN,SNRPN, UBE3A \\
\hline Infectious Disease & 4.68E-05 & GABRA5,GABRB3,GABRG3 \\
\hline Kidney Failure & 2.45E-01 & GABRB3 \\
\hline Liver Fibrosis & 1.57E-01 & NDN \\
\hline Nervous System Development and Function & $8.12 \mathrm{E}-03-1.51 \mathrm{E}-04$ & GABRA5,GABRB3,NDN, UBE3A \\
\hline Neurological Disease & 1.17E-02-5.59E-09 & GABRA5,GABRB3,GABRG3,UBE3A \\
\hline Nutritional Disease & $1.82 \mathrm{E}-03-3.03 \mathrm{E}-06$ & GABRA5,GABRB3,GABRG3 \\
\hline Organ Morphology & $5.87 \mathrm{E}-03-2.20 \mathrm{E}-03$ & GABRA5,GABRB3, UBE3A \\
\hline Organismal Functions & 5.87E-03 & NDN \\
\hline Organismal Injury and Abnormalities & $2.45 \mathrm{E}-01-3.74 \mathrm{E}-06$ & GABRA5,GABRB3,GABRG3 \\
\hline Protein Degradation & 1.33E-03 & UBE3A \\
\hline Protein Synthesis & 1.33E-03 & UBE3A \\
\hline Psychological Disorders & $5.57 \mathrm{E}-04-1.34 \mathrm{E}-06$ & GABRA5,GABRB3,GABRG3,NDN \\
\hline Renal and Urological Disease & 2.45E-01 & GABRB3 \\
\hline Reproductive System Development & 4.07E-03 & UBE3A \\
\hline Reproductive System Disease & $8.57 \mathrm{E}-03-5.87 \mathrm{E}-03$ & MKRN3, UBE3A \\
\hline Respiratory Disease & 1.07E-02-5.97E-04 & GABRA5,GABRB3,GABRG3, MKRN3, SNRPN \\
\hline Skeletal and Muscular Disorders & $8.12 \mathrm{E}-03-1.40 \mathrm{E}-08$ & GABRA5,GABRB3,GABRG3 \\
\hline Tissue Morphology & $5.26 \mathrm{E}-06-8.57 \mathrm{E}-03$ & GABRA5,GABRB3,UBE3A \\
\hline
\end{tabular}


Table 3 Diseases or functions associated with the networks constructed from genes in the amplified region

\begin{tabular}{|c|c|c|c|}
\hline Diseases or functions annotation & $p$-values & Molecules & \# molecules \\
\hline Prader-Willi syndrome & $4.69 \mathrm{E}-10$ & GABRG3,MAGEL2,MKRN3,NDN,SNRPN & 5 \\
\hline Absence seizure & 5.59E-09 & GABRA5,GABRB3, GABRG1*,GABRG3,UBE3A & 5 \\
\hline Tonic-clonic seizure & 2.07E-07 & GABRA5,GABRB3, GABRG $1 *$,GABRG3,UBE3A & 5 \\
\hline Multiple congenital anomalies & $4.32 \mathrm{E}-06$ & GABRB3,GABRG3,MAGEL2,MKRN3,NDN,PEX10*,SNRPN,UBE3A & 8 \\
\hline Stroke & $3.14 \mathrm{E}-05$ & GABRA5,GABRB3,GABRG ${ }^{*}$,GABRG3,TP53* & 5 \\
\hline Epileptic seizure & $5.60 \mathrm{E}-05$ & GABRA5,GABRB3,GABRG1*,GABRG3,UBC* & 5 \\
\hline Major depression & $1.52 \mathrm{E}-04$ & GABRA5,GABRB3, GABRG1*,GABRG3,IBTK* & 5 \\
\hline Amyotrophic lateral sclerosis & 3.77E-04 & GABRA5,GABRB3, GABRG1*,GABRG3,TP53* & 5 \\
\hline Schizophrenia & 4.37E-04 & AP1G1*,GABRA5,GABRB3, GABRG1*,GABRG3,NDN, TP53* & 7 \\
\hline Parkinson's disease & 5.57E-04 & GABRA5,GABRB3, GABRG1*,GABRG3, TP53* & 5 \\
\hline Seizures & $6.96 \mathrm{E}-04$ & GABRA5,GABRB3, GABRG1*,GABRG3,UBC*,UBE3A & 6 \\
\hline Congenital anomaly of skeletal bone & $1.40 \mathrm{E}-03$ & GABRA5,GABRB3, GABRG $1^{*}$, GABRG3, TP53* & 5 \\
\hline Weight gain & $1.82 \mathrm{E}-03$ & AP1G1*,GABRA5,GABRB3, GABRG1*,GABRG3 & 5 \\
\hline Bleeding & $9.51 \mathrm{E}-03$ & GABRA5,GABRB3, GABRG1*,GABRG3,TP53* & 5 \\
\hline
\end{tabular}

Notes: Table includes networks with at least five molecules.

*Molecules encoded by genes mapped to regions outside $15 q 11-13$.

which occurred during the anaphase stage of mitosis. The break would also have resulted in the loss of one of the two duplicated regions in some cells, giving rise to the mosaicism observed. As karyotype analysis was only done for cells from peripheral blood culture, the ratio of the two marker chromosomes in other tissues is not known. At the time of the blood sampling for genetic investigation, the patient was only 9 months old. Due to the young age when the chromosomal studies were done and the lack of data on the mosaicism level in other tissues, it is difficult to predict the course of disease manifestation and make genotype-phenotype correlation [27].

Genetic analysis determined that the genes within the regions that were duplicated in one or both of the patients are significantly associated with identified functional networks. The top three functional networks based on levels of statistical significance are developmental disorder, hereditary disorder, and neurological disease. The important genes that are involved in developmental and neurological disorders are GABRA5, GABRA3, GABRG3, MAGEL2, $M K R N 3, N D N, S N R P N$ and $U B E 3 A$. Three of the genes encode subunits of the GABA receptors, a family of ligand-gated chloride channels which mediate the major inhibitory neurotransmitter GABA in the brain. They have been found to be highly expressed in the cerebral cortex of postmortem brain samples [28]. One study found that duplications that involve this GABA gene cluster are highly enriched in an autism cohort, while another study found no difference from controls [16,29]. The imprinted gene $U B E 3 A$ functions as a transcriptional co-activator and also as a ligase in the ubiquitin proteasome pathway. $S N R P N$ also has dual functions. It is involved in RNA processing and is also spliced into several regulatory RNAs.
The remaining three genes are causative of Prader-Willi syndrome if a deletion or mutation is of paternal origin. All are intronless, transcribed only from paternal alleles and are involved in growth regulation or transcription. In addition, there are a number of C/D box snoRNAs which occur in multiple tandem copies [30]. These include the SNORD 115 (HBII-52), SNORD 116 (HBII85) and SNORD 109A (HBII-438A) clusters. They are involved in directing alternative splicing or site-specific methylation of substrate RNAs [31,32]. However, it is unclear whether they are important in cases of maternal duplications (such as the two cases in this report) as they were reported to be expressed from the paternal chromosome only [33].

\section{Conclusions}

We report two new cases of trisomy and mosaic tetrasomy 15q11-q13 of probable maternal origin from the methylation pattern. The copy number gain of genes in the region could explain the patients' phenotypic presentations. Pathway analysis identified multiple networks of candidate gene interactions. Reports of additional cases that have overlapping amplifications with different breakpoints would be helpful toward delineating the spectrum of phenotypic features and long-term follow-up for carriers of such amplifications, such as the development of late-onset Lennox-Gastaut syndrome [34] and sudden unexplained deaths [5].

\section{Consent}

Written informed consent was obtained from the parents for the laboratory investigations and publication of the case report. 


\section{Methods}

\section{Karyotype analysis}

Chromosome analyses were performed on GTG banded metaphases obtained from cultures of phytohaemagg lutinin-stimulated lymphocytes using standard methods. High-resolution chromosomes were obtained by Methotrexate cell synchronization [35].

\section{Fluorescence in situ hybridization}

Targeted cytogenetic analysis was performed on metaphase spreads using bacterial artificial chromosome probes obtained from The Hospital for Sick Children (Toronto, Canada). Slides were counterstained with 4,6-diamidino-2-phenylindole in Vectashield mounting medium (Vector Laboratories, Inc, USA) and analyzed using a fluorescence microscope Olympus BX51 equipped with a CCD Progressive Scan Video Camera (Japan Analytical Industry, Co. Ltd., Japan). Image analysis was carried out with Cytovision software (version 3.93.2) (Applied Imaging Corp, USA).

\section{Array-based copy number analysis}

Genomic DNA was extracted from peripheral blood using the Puregene DNA Isolation Kit (Qiagen GmbH, Hilden, Germany). Human CGH array consisting of $400 \mathrm{~K} 60$-mer oligonucletode probes from Agilent (Agilent Technologies Inc., Santa Clara, CA, USA) - and the reference used was human genomic DNA from Promega matched to the gender of the patient (Promega Corp., Madison, WI, USA). Test DNA was labeled with Cy5-dUTP and reference DNA was labeled with Cy3-dUTP (Sigma-Aldrich, St. Louis, MO, USA) according to Agilent's protocol for enzymatic labeling (Version 6.3). The efficiency of the labeling was measured using a Nanodrop Spectrophotometer. The labeled reference and test DNA samples were hybridized to the array at $65^{\circ} \mathrm{C}$ in an Agilent Hybridization Oven for 40 hours, with the rotator set at 20 rotations per minute. The array was then processed according to the manufacturer's instructions and scanned with an Agilent G2505C Microarray scanner at 5 micron resolution. Data were extracted from the scanned image using Agilent Feature Extraction (Version 10.7.31) and were analyzed for copy number change using Agilent Genomic Workbench Lite (Edition 6.0.130.24). Genomic coordinates are based on genome build $36 /$ hg18.

\section{qRT-PCR analysis}

Gene copy number was also investigated by relative qRT-PCR with SYBR Green dye and SNRPN as the target for quantifying copy number. Primers were designed using Primer Express (Version 3.0), and the experiment was carried out in triplicate. Genomic DNA from the two patients and a control participant was amplified in the same experiment using ZNF80 as the internal reference
[36]. Amplification was done using the Applied Biosystems StepOnePlus real time PCR system (Applied Biosystems Incorporated, Foster City, CA, USA). Results were analyzed using Applied Biosystems StepOne software (version 2.1).

\section{Methylation analysis}

Methylation status was investigated by treating the DNA with sodium bisulfite using the EpiTect Bisulfite Kit (Qiagen GmbH, Hilden, Germany), followed by qRTPCR with separate primers targeting methylated and unmethylated SNRPN according to Kubota et al. [37].

\section{Pathway analysis}

The coordinates of the minimum deleted region for $\mathrm{Pa}$ tient 2 was searched against the Human reference genome (hg18) for known genes in the region. The resulting list of genes was imported into IPA software (Ingenuity Systems, Inc. Redwood City, CA, USA) using the Entrez ID mapped to the Ingenuity Pathway Knowledge Base identifier. The reference set used was Ingenuity Knowledge Base (Genes only); relationship to include was both direct and indirect. The analysis included endogenous chemicals, and the filter summary was set to consider only relationships in which confidence $=$ experimentally observed. The statistical significance for the enrichment of genes of interest in each pathway was evaluated using a Fisher Exact test under the Core Analysis function of IPA.

\section{Abbreviations}

BP: Breakpoint; bp: Basepairs; CGH: Comparative genomic hybridization; FISH: Fluorescent in situ hybridization; GABA: Gamma aminobutyric acid; IPA: Ingenuity pathway analysis; Mb: Million base-pairs; OMIM: Online Mendelian inheritance in man; qRT-PCR: Quantitative real-time polymerase chain reaction; SSMC: Supernumerary marker chromosome; SNRPN: Small nuclear ribonucleoprotein polypeptide $\mathrm{N}$.

\section{Competing interests}

ZHL is an employee of Genomax Technologies Pte Ltd, distributor of Agilent products in Singapore. There is no competing interest for the other authors.

\section{Authors' contributions}

ECT conceived the project, obtained the funding, and participated in writing the first draft of the manuscript and in performing the array-CGH analysis; EST did the clinical assessments and participated in writing the first draft of the manuscript; ECPL performed the array-CGH and qRT-PCR experiments; $\mathrm{MHY}$ oversaw the karyotyping and performed the FISH analysis; ZHL did the pathway analysis; MSB participated in performing the array-CGH analysis. All authors read and approved the manuscript.

\section{Authors' information}

EST: Senior Consultant, Genetics Service, KK Women's and Children's Hospital, Singapore. MHY: Principal Scientific Officer, Cytogenetics Laboratory, KK Women's and Children's Hospital. ECPL: Senior Medical Technologist, KK Women's and Children's Hospital. ZHL: Application Manager (Bioinformatics), Genomax Technologies, Singapore. MSYB: Principal Scientist, KK Research Centre, KK Women's and Children's Hospital. ECT: Principal Scientist, KK Research Centre, KK Women's and Children's Hospital and Adjunct Associate Professor, Office of Clinical Sciences, Duke-NUS Graduate Medical School Singapore, Singapore. 


\section{Acknowledgments}

This work was supported by BMRC 06/1/50/19/485 (Agency for Science and Technology and Research) and NMRC/PPG/KKH12010-Theme3 (National Medical Research Council, Ministry of Health, Republic of Singapore). The authors appreciate the medical editing assistance of Jon Kilner, MS, MA (Pittsburgh, Pennsylvania, USA).

\section{Author details}

${ }^{1}$ Genetics Service, KK Women's \& Children's Hospital, 100 Bukit Timah Road 229899 Singapore, Singapore. ${ }^{2}$ Cytogenetics Laboratory, KK Women's \& Children's Hospital, 100 Bukit Timah Road 229899 Singapore, Singapore. ${ }^{3}$ KK Research Laboratory, KK Women's \& Children's Hospital, 100 Bukit Timah Road 229899 Singapore, Singapore. ${ }^{4}$ Genomax Technologies Pte Ltd, 51 Science Park Road, \#04-15 117586 Singapore, Singapore. ${ }^{5}$ Office of Clinical Sciences, Duke-NUS Graduate Medical School, 8 College Road 169857 Singapore, Singapore.

Received: 27 February 2014 Accepted: 11 April 2014

Published: 16 May 2014

\section{References}

1. Pujana MA, Nadal M, Guitart M, Armengol L, Gratacos M, Estivill X: Human chromosome 15q11-q14 regions of rearrangements contain clusters of LCR15 duplicons. Eur J Hum Genet 2002, 10:26-35.

2. Saadallah N, Hulten M: Chiasma distribution, genetic lengths, and recombination fractions: a comparison between chromosomes 15 and 16. J Med Genet 1983, 20:290-299.

3. Cassidy SB, Schwartz S, Miller JL, Driscoll DJ: Prader-Willi syndrome. Genet Med 2012, 14:10-26.

4. Battaglia A, Parrini B, Tancredi R: The behavioral phenotype of the idic(15) syndrome. Am J Med Genet C: Semin Med Genet 2010, 154C:448-455.

5. Battaglia A: The inv dup (15) or idic (15) syndrome (Tetrasomy $15 q$ ). Orphanet J Rare Dis 2008, 3:30.

6. Wang NJ, Liu D, Parokonny AS, Schanen NC: High-resolution molecular characterization of 15q11-q13 rearrangements by array comparative genomic hybridization (array CGH) with detection of gene dosage. Am J Hum Genet 2004, 75:267-281.

7. Yang J, Yang Y, Huang Y, Hu Y, Chen X, Sun H, Lv Z, Cheng Q, Bao L: A study of two Chinese patients with tetrasomy and pentasomy $15 q 11 q 13$ including Prader-Willi/Angelman syndrome critical region present with developmental delays and mental impairment. BMC Med Genet 2013, 14:9.

8. Browne CE, Dennis NR, Maher E, Long FL, Nicholson JC, Sillibourne J, Barber JC: Inherited interstitial duplications of proximal 15q: genotype-phenotype correlations. Am J Hum Genet 1997, 61:1342-1352.

9. Cai G, Edelmann L, Goldsmith JE, Cohen N, Nakamine A, Reichert JG, Hoffman EJ, Zurawiecki DM, Silverman JM, Hollander E, Soorya L, Anagnostou E, Betancur C, Buxbaum JD: Multiplex ligation-dependent probe amplification for genetic screening in autism spectrum disorders: efficient identification of known microduplications and identification of a novel microduplication in ASMT. BMC Med Genomics 2008, 1:50.

10. Kitsiou-Tzeli S, Tzetis M, Sofocleous C, Vrettou C, Xaidara A, Giannikou K, Pampanos A, Mavrou A, Kanavakis E: De novo interstitial duplication of the 15q11.2-q14 PWS/AS region of maternal origin: Clinical description, array CGH analysis, and review of the literature. Am J Med Genet A 2010, 152A:1925-1932.

11. Crespi BJ, Crofts HJ: Association testing of copy number variants in schizophrenia and autism spectrum disorders. J Neurodev Disord 2012, 4:15.

12. de Kovel CG, Trucks H, Helbig I, Mefford HC, Baker C, Leu C, Kluck C, Muhle H, von Spiczak S, Ostertag P, Obermeier T, Kleefuss-Lie AA, Hallmann K, Steffens M, Gaus V, Klein KM, Hamer HM, Rosenow F, Brilstra EH, Trenite DK, Swinkels ME, Weber YG, Unterberger I, Zimprich F, Urak L, Feucht M, Fuchs K, Moller RS, Hjalgrim H, De Jonghe $P$, et al: Recurrent microdeletions at $15 \mathrm{q} 11.2$ and $16 \mathrm{p} 13.11$ predispose to idiopathic generalized epilepsies. Brain 2010, 133:23-32.

13. Depienne C, Moreno-De-Luca D, Heron D, Bouteiller D, Gennetier A, Delorme R, Chaste P, Siffroi JP, Chantot-Bastaraud S, Benyahia B, Trouillard O, Nygren G, Kopp S, Johansson M, Rastam M, Burglen L, Leguern E, Verloes A, Leboyer M, Brice A, Gillberg C, Betancur C: Screening for genomic rearrangements and methylation abnormalities of the 15q11-q13 region in autism spectrum disorders. Biol Psychiatry 2009, 66:349-359.

14. Stewart LR, Hall AL, Kang SH, Shaw CA, Beaudet AL: High frequency of known copy number abnormalities and maternal duplication 15q11-q13 in patients with combined schizophrenia and epilepsy. BMC Med Genet 2011, 12:154.

15. Vorstman JA, Staal WG, van Daalen E, van Engeland $H$, Hochstenbach PF, Franke $L$ : Identification of novel autism candidate regions through analysis of reported cytogenetic abnormalities associated with autism. Mol Psychiatry 2006, 11:1. 18-28.

16. Matsunami N, Hadley D, Hensel CH, Christensen GB, Kim C, Frackelton E, Thomas K, da Silva RP, Stevens J, Baird L, Otterud B, Ho K, Varvil T, Leppert T, Lambert CG, Leppert M, Hakonarson H: Identification of rare recurrent copy number variants in high-risk autism families and their prevalence in a large ASD population. PLOS One 2013, 8:e52239.

17. Bassett AS, Scherer SW, Brzustowicz LM: Copy number variations in schizophrenia: critical review and new perspectives on concepts of genetics and disease. Am J Psychiatry 2010, 167:899-914.

18. Hosak L, Silhan P, Hosakova J: Genomic copy number variations: a breakthrough in our knowledge on schizophrenia etiology? Neuro Endocrinol Lett 2012, 33:183-190.

19. Miller DT, Shen Y, Weiss LA, Korn J, Anselm I, Bridgemohan C, Cox GF, Dickinson H, Gentile J, Harris DJ, Hegde V, Hundley R, Khwaja O, Kothare S, Luedke C, Nasir R, Poduri A, Prasad K, Raffalli P, Reinhard A, Smith SE, Sobeih MM, Soul JS, Stoler J, Takeoka M, Tan WH, Thakuria J, Wolff R, Yusupov R, Gusella JF, et al: Microdeletion/duplication at 15q13.2q13.3 among individuals with features of autism and other neuropsychiatric disorders. J Med Genet 2009, 46:242-248.

20. Moreno-De-Luca D, Sanders SJ, Willsey AJ, Mulle JG, Lowe JK, Geschwind $\mathrm{DH}$, State MW, Martin CL, Ledbetter DH: Using large clinical data sets to infer pathogenicity for rare copy number variants in autism cohorts. Mol Psychiatry 2013, 18:1090-1095.

21. Urraca N, Cleary J, Brewer V, Pivnick EK, McVicar K, Thibert RL, Schanen NC, Esmer C, Lamport D, Reiter LT: The Interstitial Duplication 15q11.2-q13 Syndrome includes Autism, mild facial anomalies and a characteristic EEG Signature. Autism Res 2013, 6:268-279.

22. Burnside RD, Pasion R, Mikhail FM, Carroll AJ, Robin NH, Youngs EL, Gadi IK, Keitges E, Jaswaney VL, Papenhausen PR, Potluri VR, Risheg H, Rush B, Smith JL, Schwartz S, Tepperberg JH, Butler MG: Microdeletion/microduplication of proximal 15q11.2 between BP1 and BP2: a susceptibility region for neurological dysfunction including developmental and language delay. Hum Genet 2011, 130:517-528.

23. Tatton-Brown K, Pilz DT, Orstavik KH, Patton M, Barber JC, Collinson MN, Maloney VK, Huang S, Crolla JA, Marks K, Ormerod E, Thompson P, Nawaz Z, Lese-Martin C, Tomkins S, Waits P, Rahman N, McEntagart M: 15q overgrowth syndrome: a newly recognized phenotype associated with overgrowth, learning difficulties, characteristic facial appearance, renal anomalies and increased dosage of distal chromosome 15q. Am J Med Genet A 2009, 149A:147-154

24. Al Ageeli E, Drunat S, Delanoe C, Perrin L, Baumann C, Capri Y, Fabre-Teste J, Aboura A, Dupont C, Auvin S, El Khattabi L, Chantereau D, Moncla A, Tabet AC, Verloes A: Duplication of the 15q11-q13 region: clinical and genetic study of 30 new cases. Eur J Med Genet 2014, 57:5-14

25. Hogart A, Leung KN, Wang NJ, Wu DJ, Driscoll J, Vallero RO, Schanen NC, LaSalle JM: Chromosome 15q11-13 duplication syndrome brain reveals epigenetic alterations in gene expression not predicted from copy number. J Med Genet 2009, 46:86-93.

26. Wandstrat AE, Schwartz S: Isolation and molecular analysis of inv dup(15) and construction of a physical map of a common breakpoint in order to elucidate their mechanism of formation. Chromosoma 2000, 109:498-505.

27. Biesecker LG, Spinner NB: A genomic view of mosaicism and human disease. Nat Rev Genet 2013, 14:307-320.

28. Hogart A, Nagarajan RP, Patzel KA, Yasui DH, Lasalle JM: 15q11-13 GABAA receptor genes are normally biallelically expressed in brain yet are subject to epigenetic dysregulation in autism-spectrum disorders. Hum Mol Genet 2007, 16:691-703.

29. Hedges DJ, Hamilton-Nelson KL, Sacharow SJ, Nations L, Beecham GW, Kozhekbaeva ZM, Butler BL, Cukier HN, Whitehead PL, Ma D, Jaworski JM, Nathanson L, Lee JM, Hauser SL, Oksenberg JR, Cuccaro ML, Haines JL, Gilbert JR, Pericak-Vance MA: Evidence of novel fine-scale structural variation at autism spectrum disorder candidate loci. Mol Autism 2012, 3:2. 
30. Sridhar P, Gan HH, Schlick T: A computational screen for C/D box snoRNAs in the human genomic region associated with Prader-Willi and Angelman syndromes. J Biomed Sci 2008, 15:697-705.

31. Galardi S, Fatica A, Bachi A, Scaloni A, Presutti C, Bozzoni I: Purified box C/D snoRNPs are able to reproduce site-specific 2'-O-methylation of target RNA in vitro. Mol Cell Biol 2002, 22:6663-6668.

32. Kishore S, Khanna A, Zhang Z, Hui J, Balwierz PJ, Stefan M, Beach C, Nicholls RD, Zavolan M, Stamm S: The snoRNA MBII-52 (SNORD 115) is processed into smaller RNAs and regulates alternative splicing. Hum Mol Genet 2010, 19:1153-1164.

33. Runte $M$, Varon R, Horn D, Horsthemke B, Buiting K: Exclusion of the C/D box snoRNA gene cluster HBII-52 from a major role in Prader-Willi syndrome. Hum Genet 2005, 116:228-230

34. Orrico A, Zollino M, Galli L, Buoni S, Marangi G, Sorrentino V: Late-onset Lennox-Gastaut syndrome in a patient with 15q11.2-q13.1 duplication. Am J Med Genet A 2009, 149A:1033-1035.

35. Yunis JJ: High resolution of human chromosomes. Science 1976, 191:1268-1270.

36. Hoebeeck J, van der Luijt R, Poppe B, De Smet E, Yigit N, Claes K, Zewald R, de Jong GJ, De Paepe A, Speleman F, Vandesompele J: Rapid detection of VHL exon deletions using real-time quantitative PCR. Lab Invest 2005, 85:24-33.

37. Kubota T, Das S, Christian SL, Baylin SB, Herman JG, Ledbetter DH: Methylation-specific PCR simplifies imprinting analysis. Nat Genet 1997, 16:16-17.

doi:10.1186/1755-8166-7-32

Cite this article as: Tan et al:: Chromosome 15q11-q13 copy number gain detected by array-CGH in two cases with a maternal methylation pattern. Molecular Cytogenetics 2014 7:32.

\section{Submit your next manuscript to BioMed Central and take full advantage of:}

- Convenient online submission

- Thorough peer review

- No space constraints or color figure charges

- Immediate publication on acceptance

- Inclusion in PubMed, CAS, Scopus and Google Scholar

- Research which is freely available for redistribution 